\title{
Diversidade florística do estrato arbustivo-arbóreo em quintais agroflorestais do reassentamento Mariana, TO
}

\author{
Ícaro Gonçalves Santos ${ }^{1 *}$, Enderson Alves Nunes ${ }^{1}$, Priscila Bezerra de Souza' ${ }^{1}$, Conceição Aparecida Previero² \\ ${ }^{1}$ Universidade Federal do Tocantins, Rua Badejós, Chácaras 69 e 72, CP. 66, CEP 77402-970, Gurupi, TO, Brasil \\ ${ }^{2}$ Universidade Luterana do Brasil, Av. Teotônio Segurado ACSU-SO 150, Plano Diretor Sul, CEP 77054970, Palmas, TO, Brasil.
}

"Autor correspondente:
icaro.gbio@gmail.com

Termos para indexação:

Biodiversidade

Heterogeneidade

Dominância

Index terms:

Biodivesity

Genetic heterogeneity

Dominance

Histórico do artigo:

Recebido em 19/02/2017

Aprovado em 17/12/2017

Publicado em 29/12/2017

doi: $10.4336 / 2017 . p f b .37 .92 .1412$

\begin{abstract}
Resumo - Objetivou-se analisar a florística, diversidade e equabilidade do estrato arbustivo-arbóreo de quatro quintais agroflorestais (QAs), no Reassentamento Mariana, localizado entre os municípios de Palmas e Porto Nacional no estado do Tocantins. Foram instaladas três parcelas de $20 \times 30 \mathrm{~m}$ em cada QA, perfazendo um total de 0,72 ha de área amostral. Foram inventariados todos os indivíduos arbustivo-arbóreos com circunferência a $1,30 \mathrm{~m}$ do solo $(\mathrm{CAP}) \geq 10 \mathrm{~cm}$. Nos quatro QAs foram verificados 477 indivíduos e 81 espécies pertencentes a 34 famílias e 73 gêneros. $O$ índice de diversidade de Shannon (H') foi 3,68 e o índice de equabilidade de Pielou (J'), 0,83 para a área total amostrada. Individualmente, os quintais agroflorestais apresentaram $H^{\prime}$ $=2,52 ; 3,27 ; 2,66$ e 2,94, e J'=0,78; 0,90; 0,77 e 0,85 para QA1, QA2, QA3 e QA4, respectivamente. Pode-se inferir que os quintais estudados apresentam alta riqueza e diversidade, evidenciando grande heterogeneidade ambiental e baixa dominância ecológica.
\end{abstract}

\section{Floristic diversity of the shrub-arboreal stratum of homegardens in the Mariana re-settlement, Tocantins State, Brazil}

\begin{abstract}
The objective was to analyze the floristic, diversity and equability of the tree shrub stratum of home gardens (QA) in Mariana re-settlement located between Palmas and Porto Nacional municipalities in Tocantins State, Brazil. Three $20 \times 30 \mathrm{~m}$ plots were installed in each home garden, totalizing 0.72 ha of sampled area. All shrub-tree individuals had its circumference at $1.3 \mathrm{~m}$ above ground level $(\mathrm{CBH})$ measured when $\mathrm{CBH} \geq 10 \mathrm{~cm}$. A total of 477 individuals, 81 species, 34 families and 73 genera were verified in all QA. Shannon diversity index $\left(\mathrm{H}^{\prime}\right)$ was 3.68 and Pielou equability indice (J') was 0.83 , for all sampled area. Individually, QA1, QA2, QA3 and QA4 presented $\mathrm{H}^{\prime}=2.52 ; 3.27 ; 2.66$ and 2.94 , and $\mathrm{J}^{\prime}=0.78 ; 0.90 ; 0.77$ and 0.85 , respectively. It was possible to infer that the studied QA showed high richness and diversity, evidencing great environmental heterogeneity and low ecological dominance.
\end{abstract}

\section{Introdução}

O bioma Cerrado possui uma área de 2,04 milhões de $\mathrm{km}^{2}$, o que equivale a aproximadamente $22 \%$ do território nacional sendo, considerado o segundo maior bioma brasileiro (Finger \& Finger, 2015; Silva \& Sano, 2016).
As áreas de Cerrado no território brasileiro vêm diminuindo ao passar dos anos, conforme os investimentos para a expansão do agronegócio e pecuária aumentam (Sano, 2010). Além desses dois fatores, a extensão urbana e o aumento da população humana acabam sendo 
responsáveis por aberturas de novas áreas e mudanças do uso da terra, a fim de subsidiar as necessidades básicas das civilizações (Silva \& Sano, 2016).

Uma das necessidades de abertura de novas áreas, por meio do desmatamento do bioma Cerrado, é para as construções de usinas hidrelétricas. Esses empreendimentos são os principais responsáveis pela produção energética do País; contrapondo-se a esse benefício, as hidrelétricas são consideradas como empreendimentos de grande impacto ambiental, por modificar fisicamente grandes extensões de áreas, o que influencia nas mudanças da biota envolvida, bem como as comunidades ribeirinhas e adjacentes (Evans et al., 2009).

No Estado do Tocantins, a primeira usina hidrelétrica de grande porte teve seu contrato de concessão firmado em 1997 pela empresa INVESTCO S.A., sendo sua construção finalizada em 2000, fechando sua primeira comporta em 2001. A área inundada pelo barramento foi equivalente a $630 \mathrm{~km}^{2}$, impactando diretamente 6.483 pessoas, as quais tiveram que ser indenizadas ou realocadas, dando início aos reassentamentos rurais nos municípios atingidos pela barragem Usina Hidrelétrica Luis Eduardo Magalhães (Parente \& Miranda, 2014).

De acordo com Leturcq (2010), os reassentamentos podem ser entendidos como uma migração induzida a qual força o abandono do espaço de vida dos indivíduos para um novo ambiente onde não existe uma identidade. Segundo Batista (2009), o ato de reassentar coletivamente as populações impactadas pode ser considerado como uma das ações que mais se aproximam dos pré-requisitos, os quais garantiriam um restabelecimento adequado das condições de vida dessa população, o que possibilitaria que os impactados com maior laço social pudessem compartilhar o espaço com um formato próximo ao de origem. Porém, é necessário que esses reassentamentos sejam planejados de maneira que assegurem que essa população tenha condições mínimas, ferramentas, apoio técnico e social para que consigam se adaptar a esse novo ambiente e possam tornar essa relação produtiva.

Com a criação desses reassentamentos, houve mudanças no uso da terra e, consequentemente, nas características naturais do Cerrado, onde os proprietários (reassentados) definiam suas áreas de uso, como pastagem e lavoura, além de delimitarem as áreas referentes a suas moradias, bem como os quintais associados a essas residências (Santos et al., 2016).

Pesq. flor. bras., Colombo, v. 37, n. 92, p. 513-524, out./dez. 2017
Conforme Chagas et al. (2012), uma dos atributos que devem ser levados em consideração, na avaliação do desenvolvimento da adaptação das famílias reassentadas, é a maneira como essas pessoas se relacionam com o meio ambiente e como as famílias agricultoras manejam os agroecossistemas. Um dos modelos sustentáveis de uso e manejo da terra por essas famílias são os quintais agroflorestais (QAs).

Os quintais agroflorestais são áreas de produção próximas às residências, onde se cultivam diversas espécies medicinais, florestais, agrícolas e ornamentais, sendo esses espaços considerados fundamentais para o lazer e para a subsistência das famílias proprietárias (Brizídio \& Nunes, 2010). Essas áreas são consideradas como reservatórios da diversidade de espécies vegetais, as quais são manejadas pelas famílias. São mantidas por diversas razões, sendo a principal delas a garantia da segurança alimentar das famílias proprietárias através da produção de frutos para a alimentação (Nair, 1986; Kumar et al., 1994; Rocha Garcia et al., 2015).

Estudos sobre a diversidade florística são de grande importância para a compreensão dos quintais agroflorestais, por abordarem dados ecológicos como a florística e a fitossociologia; o que permite o entendimento da estrutura das comunidades, suas interações ecológicas, bem como a identificação de espécies predominantes. Estes dados são importantes norteadores para a elaboração de planos de manejo, conservação das espécies vegetais e preservação ambiental (Vieira et al., 2012).

No Estado do Tocantins, estudos fitotossociológicos nos quintais agroflorestais são praticamente inexistentes, o que acarreta em uma lacuna de informações cientificas e técnicas sobre essas áreas. Mediante isso, esse estudo teve por objetivo contribuir para o conhecimento da composição, dinâmica e estrutura florística dos quintais agroflorestais do reassentamento Mariana, bem como gerar informações socioambientais dos reassentados, no sentido de perceber a resiliência desses proprietários no que concerne ao pertencimento da terra e a interação agroecologica dos reassentados com os quintais agroflorestais (QAs).

\section{Material e métodos}

\section{Caracterização da área de estudo}

O estudo foi realizado no reassentamento Mariana, o qual foi implantado no segundo semestre de 2001, 
devido à realocação dos ribeirinhos impactados pelo enchimento da barragem da Usina Hidrelétrica Luis Eduardo Magalhães.

Antes do realocamento dos ribeirinhos atingidos pela barragem da usina, a área onde hoje é o reassentamento Mariana foi comprada pela empresa responsável pela usina. A escolha do local foi definida em conjunto com os reassentados, sendo esse local uma antiga fazenda de lavoura de arroz.

O reassentamento está localizado entre os municípios de Palmas e Porto Nacional, TO, ambos inseridos no bioma Cerrado. A coordenada geográfica da sede da associação dos reassentados de Mariana é $10^{\circ} 24^{\prime} 2,61^{\prime \prime} S$ e 48¹0'19,06”'W.

O reassentamento Mariana foi escolhido como objeto de estudo, tanto pela localização quanto pelo histórico de aceitação dos proprietários para a realização de pesquisas cientificas com os autores deste estudo. Além de contar com uma associação, que é determinante para a organização participativa dos moradores e, consequentemente, para a apresentação de informações.

O clima da região é do tipo AW, segundo Köppen, sendo definido como tropical úmido, com estação chuvosa no verão e seca no inverno, tendo temperatura média ao longo do ano entre $22{ }^{\circ} \mathrm{C}$ e $28^{\circ} \mathrm{C}$ (Klink \& Machado, 2005). Os municípios possuem altitude média de $212 \mathrm{~m}$ e precipitação média anual de $1.622 \mathrm{~mm}$ (Tocantins, 2012). Os solos da região são classificados como Latossolo Roxo e Latossolo Vermelho-Escuro.

$\mathrm{O}$ reassentamento Mariana possui uma área total de 361,9 ha, sendo $35 \%$ destinado à reserva legal. A área restante foi, inicialmente, dividida em 14 lotes (14 famílias) de 4,2 ha a 30 ha, conforme o acordo de indenização de cada impactado. Atualmente, alguns lotes foram revendidos e não pertencem mais aos impactados pelo reassentamento (Santos, 2009; Parente \& Miranda, 2014).

\section{Seleção e delimitação dos quintais agroflorestais (QAs)}

A seleção das unidades de produção familiar foi realizada através de metodologia não probabilística, considerando que as famílias selecionadas deviam ter sido impactadas pelo represamento da Usina Hidrelétrica Luis Eduardo Magalhães e tinham que participar ativamente das pesquisas de campo, uma vez que os mesmos têm papel fundamental no apontamento do uso e da estrutura dos quintais.
Após a triagem, os quintais agroflorestais com as características desejadas para a pesquisa foram eleitos a partir da técnica conhecida como "bola de neve" (snowball), sendo esta uma amostragem em cadeia de referência. Inicialmente, foi eleita como informante a presidente da associação do reassentamento Mariana, que indicou outra família reassentada, essa segunda família indicou uma terceira e assim por diante. Desta forma, as indicações fornecidas pelos proprietários se repetiram e totalizaram quatro quintais indicados (Albuquerque et al., 2010; Pinto, 2012).

Para realizar a amostragem florística, foi necessário demarcar os lotes e parcelas. Desta forma, utilizou-se os softwares Google Earth e ArcGis 10.2 Desktop. Com o Google Earth obteve-se imagens de satélite de alta resolução espacial do sensor Image (C) 2015 DigitalGlobe, correspondente ao ano de 2015, com composição colorida pré-definida (RGB). O perímetro do reassentamento Mariana foi adquirido através da empresa administradora da Usina Hidrelétrica Luis Eduardo Magalhães e responsável pelos reassentamentos, INVESTCO S.A. O arquivo foi obtido em formato ESRI-Shape. O perímetro do imóvel foi utilizado para o georreferenciamento das imagens de satélite do Google Earth, onde se utilizou o software de SIG ArcGIS 10.2 Desktop. Dessa forma, as imagens foram georeferenciadas a partir dos pontos de controle do mapa do reassentamento através da ferramenta "Georreferencing" (Freitas et al., 2012).

\section{Coleta de dados}

Foi realizado inventário florestal em quatro quintais agroflorestais (QA) inseridos na fisionomia cerrado sensu stricto, sendo adotado o método de amostragem de área fixa; a vegetação do componente arbustivoarbóreo foi avaliada quantitativamente pelo método de parcelas (Gazel Filho, 2008; Miguel et al., 2016). Foram instaladas três parcelas amostrais em QA, com dimensões de $20 \mathrm{~m}$ x $30 \mathrm{~m}$ cada, perfazendo um total de 0,72 ha. A distribuição destas parcelas ocorreu de maneira aleatória, adaptadas ao formato dos QAs, pois estes possuem diferentes tamanhos e estruturas (Pinto, 2012).

Após a distribuição das parcelas definidas no inventário florestal, foram amostrados todos os indivíduos arbustivo-arbóreos nativos ou exóticos com circunferência a $1,30 \mathrm{~m}$ do solo (CAP) $\geq 10$ $\mathrm{cm}$ (Gonçalves et al., 2015). Os indivíduos que apresentavam ramificações foram incluídos apenas 
quando pelo menos uma das ramificações obedecia ao critério de inclusão (CAP $\geq 10 \mathrm{~cm}$ ), sendo, anotado o CAP de todas as ramificações para o cálculo da área basal (Kunz et al., 2009).

Os quintais foram identificados com numeração sequencial, estando localizados em: QA1 $=10^{\circ} 24^{\prime}$ $23,43^{\prime \prime}$ S e $48^{\circ} 10^{\prime} 18,45^{\prime \prime} \mathrm{W}$; QA2 = 10²3' $59,82^{\prime}$ 'S e $48^{\circ}$

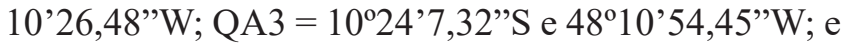
QA4 $=10^{\circ} 24^{\prime} 16,27^{\prime \prime}$ 'S e 48 ${ }^{\circ} 10^{\prime} 23,47^{\prime \prime} \mathrm{W}$.

A identificação dos indivíduos foi realizada in loco, seguindo critérios de reconhecimento das características dendrológicas, identificação por chaves de classificação e consultas por meio de comparações com literatura especializada, como Lorenzi (2002) e Silva Júnior (2012). As espécies que não foram identificadas em campo tiveram material vegetal coletado, para comparação com materiais botânicos depositados no Herbário da Universidade Federal do Tocantins Campus Porto Nacional, com auxílio de especialistas. As espécies foram classificadas com base no sistema APG III (Angiosperm Phylogeny Group, 2009). A grafia e autoria dos binômios específicos e sinonímias foram confirmadas nas bases de dados da Lista de Espécies da Flora do Brasil (Reflora, 2015).

O levantamento dos dados referente ao manejo dos quintais foi realizado através de entrevista semiestruturada com questões abertas, bem como com turnê guiada, onde os proprietários dos QAs participavam do levantamento florístico como informantes, indicando o nome popular das espécies, o uso e beneficiamento dos produtos madeireiros e não madeireiros das principais espécies utilizadas e o histórico das áreas dos QAs (Florentino et al., 2007).

\section{Diversidade}

Para obter o índice de diversidade de ShannonWeaver (H') e a equabilidade de Pielou, que representa a uniformidade (J'), utilizou-se o software Fitopac versão 2.1.2 (Sheperd, 2010). O índice de ShannonWeaver considera diferentes pesos entre as espécies raras e abundantes, ou seja, é sensível às espécies menos comuns, ou consideradas raras localmente, sendo considerado um índice não paramétrico que mede a diversidade de espécies com base no número e na abundância relativa das mesmas (Freitas \& Magalhães, 2012). O valor de J' é determinado em um intervalo de 0 a 1 , sendo que o valor máximo representa a situação em que todas as espécies possuem a mesma abundância e a maior distribuição dos indivíduos por espécie (Kunz et al., 2009; Finger \& Finger, 2015).

Uma vez identificado em levantamento bibliográfico que não há uma padronização da apresentação dos resultados obtidos em outras pesquisas com QA, optouse por apresentar os resultados, tanto em valores brutos, quanto por medidas de posição e dispersão (Gotelli \& Ellison, 2011).

\section{Resultados}

No reassentamento Mariana foram obtidos 477 indivíduos, 81 espécies, 34 famílias e 73 gêneros (Tabelas 1 e 2).

Tabela 1. Valores obtidos no inventário dos quatro quintais agroflorestais do reassentamento Mariana, TO.

\begin{tabular}{ccccc}
\hline Áreas de estudo & $\mathbf{N}^{\mathbf{0}}$ in & $\mathbf{N}^{\mathbf{0}}$ fam & $\mathbf{N}^{\mathbf{a}}$ gên & $\mathbf{N}^{\mathbf{o}} \mathbf{s p}$ \\
\hline QA 01 & 147 & 17 & 28 & 32 \\
QA 02 & 112 & 22 & 36 & 38 \\
QA 03 & 114 & 20 & 29 & 31 \\
QA 04 & 104 & 20 & 31 & 31
\end{tabular}

Em que: $\mathrm{N}^{\circ}$ in $=$ número de indivíduos, $\mathrm{N}^{\circ} \mathrm{sp}=$ número de espécies, $\mathrm{N}^{\circ}$ fam $=$ número de famílias, $\mathrm{N}^{\circ}$ gên $=$ número de gêneros .

Dos 28 gêneros do QA1, apenas três (11\%) foram representados com mais de uma espécie. (Citrus com 3 e Psidium e Theobroma com 2 espécies cada). Dos 36 gêneros amostrados, no QA2 apenas dois (6\%) foram representados por mais de uma espécie (2), sendo Byrsonima e Tabebuia. No QA3, dos 28 gêneros encontrados, apenas três (11\%) foram representados por mais de uma espécie (2), sendo Byrsonima, Citrus e Theobroma, e no QA4, nenhum dos 31 gêneros amostrados apresentou mais de uma espécie. Dentre todos os 73 gêneros identificados nos quatro QA seis (8\%) apresentaram mais de uma espécie: Byrsonima e Citrus, com 3 e Inga, Psidium, Tabebuia e Theobroma, com duas 2 espécies cada.

Muitas famílias tiveram uma só espécie como representante. As famílias mais ricas foram: no QA1 (59\%), Arecaceae (4 sp.), Anacardiaceae, Fabaceae e Rutaceae (3 sp.); no QA2 (28\%), Fabaceae (11 sp.) e Malpighiaceae (3 sp.); no QA3 (35\%), Malvaceae (4 sp.), Anacardiaceae e Malpighiaceae (3 sp.); e no QA4 (25\%), Fabaceae (5 sp.) e Malvaceae (4 sp.). Considerando os quatro quintais agroflorestais em conjunto, apenas 16 $(47 \%)$ apresentaram mais de uma espécie, sendo que as 
mais ricas foram Fabaceae (17 sp.), Malvaceae (7 sp.) e Arecaceae (6 sp.).

Das 34 famílias encontradas nos quatro quintais agroflorestais, somente sete famílias estiveram presentes em todos os QAs (Tabela 1). As famílias Anacardiaceae, Arecaceae, Fabaceae, Malpighiaceae, Malvaceae e Myrtaceae apresentam espécies frutíferas utilizadas na alimentação dos proprietários, como caju, manga, bacaba, tamarindo, entre outros, enquanto que a família Bignoniaceae foi representada por três espécies utilizadas na arborização e ornamentação dos quintais agroflorestais, devido à sua beleza cênica, a exemplo dos ipês.

Tabela 2. Lista das famílias/espécies amostradas nos quintais agroflorestais amostrados no Reassentamento Mariana, TO.

\begin{tabular}{|c|c|c|}
\hline Famílias/espécie & Nome popular & *Uso \\
\hline \multicolumn{3}{|l|}{ Anacardiaceae } \\
\hline Anacardium occidentale $\mathrm{L}$. & Caju & $\mathrm{Al}, \mathrm{Co}, \mathrm{Ar}$ \\
\hline Mangifera indica $\mathrm{L}$. & Manga & $\mathrm{Al}, \mathrm{Ar}$ \\
\hline Spondias mombin $\mathrm{L}$ & Cajá & $\mathrm{Al}, \mathrm{Co}, \mathrm{Ar}$ \\
\hline \multicolumn{3}{|l|}{ Annonaceae } \\
\hline Annona crassiflora Mart & Araticum & $\mathrm{Al}, \mathrm{Me}, \mathrm{Co}, \mathrm{Ar}$ \\
\hline $\begin{array}{c}\text { Xylopia aromatica (Lam.) } \\
\text { Mart. }\end{array}$ & Pimenta de macaco & $\mathrm{Ar}$ \\
\hline \multicolumn{3}{|l|}{ Apocynaceae } \\
\hline $\begin{array}{c}\text { Aspidosperma macrocarpon } \\
\text { Mart. }\end{array}$ & Guatambu & $\mathrm{Me}, \mathrm{Ar}$ \\
\hline \multicolumn{3}{|l|}{ Arecaceae } \\
\hline $\begin{array}{l}\text { Acrocomia aculeata (Jacq.) } \\
\text { Lodd. ex Mart. }\end{array}$ & Macaúba & $\mathrm{Al}, \mathrm{Ar}$ \\
\hline $\begin{array}{l}\text { Attalea phalerata Mart. ex } \\
\text { Spreng. }\end{array}$ & Bacuri & $\mathrm{Al}, \mathrm{Ar}$ \\
\hline Cocos nucifera $\mathrm{L}$. & Coco da Bahia & $\mathrm{Al}, \mathrm{Ar}$ \\
\hline Euterpe oleracea Mart. & Açaí & $\mathrm{Al}, \mathrm{Co}, \mathrm{Ar}$ \\
\hline Mauritia flexuosa L. f. & Buriti & $\mathrm{Al}, \mathrm{Co}, \mathrm{Ar}$ \\
\hline Oenocarpus bacaba Mart. & Bacaba & $\mathrm{Al}, \mathrm{Co}, \mathrm{Ar}$ \\
\hline \multicolumn{3}{|l|}{ Asteraceae } \\
\hline $\begin{array}{l}\text { Eremanthus erythropappus } \\
\text { (DC.) MacLeish }\end{array}$ & Candeia & Or, Ar \\
\hline \multicolumn{3}{|l|}{ Bignoniaceae } \\
\hline $\begin{array}{c}\text { Handroanthus } \\
\text { impetiginosus (Mart. ex } \\
\text { DC.) Mattos }\end{array}$ & Ipê rosa & Or, Ar \\
\hline $\begin{array}{c}\text { Tabebuia aurea (Silva } \\
\text { Manso)Benth.\& Hook.f ex } \\
\text { S. Moore }\end{array}$ & Ipê amarelo & Or, Ar \\
\hline $\begin{array}{c}\text { Tabebuia caraiba (Mart.) } \\
\text { Bureau }\end{array}$ & Caraíba & Or, Ar \\
\hline
\end{tabular}

Bixaceae

Continua...
Tabela 2. continuação.

\begin{tabular}{ccc}
\hline Famílias/espécie & Nome popular & *Uso \\
\hline Bixa orellana $\mathrm{L}$. & Urucum & $\mathrm{Me}, \mathrm{Ar}$ \\
\hline Calophyllaceae & & \\
$\begin{array}{c}\text { Calophyllum brasiliense } \\
\text { Cambess } \\
\text { Kielmeyera lathrophyton } \\
\text { Saddi }\end{array}$ & Landi & $\mathrm{Ma}, \mathrm{Ar}$ \\
$\begin{array}{c}\text { Cannabaceae } \\
\text { Trema micrantha }(\mathrm{L} .) \\
\text { Blume }\end{array}$ & Pau-santo & \\
Caricaceae & & $\mathrm{Ar}$ \\
Carica papaya $\mathrm{L}$. & & $\mathrm{Al}, \mathrm{Me}, \mathrm{Ar}$ \\
Caryocaraceae & Mamão & \\
$\begin{array}{c}\text { Caryocar brasiliense } \\
\text { Cambess. }\end{array}$ & & $\mathrm{Al}, \mathrm{Ar}$ \\
\hline
\end{tabular}

Chrysobalanaceae

Licania tomentosa (Benth.)
Fritsch

Combretaceae

Buchenavia tomentosa Eichler

Tarumã

$\mathrm{Ar}$

Terminalia catappa $\mathrm{L}$.

Amendoeira da praia $\mathrm{Al}, \mathrm{Ar}$

\section{Connaraceae}

Connarus suberosus Planch.

- $\mathrm{Ar}$ Dilleniaceae

$\begin{array}{ccc}\text { Curatella americana } \mathrm{L} . & \text { Lixeira } & \mathrm{Ar} \\ \text { Mouriri pusa Gardner } & \text { Puçá } & \mathrm{Ar}\end{array}$

Ebenaceae

Diospyros hispida A. DC. Olho de boi Ar

Euphorbiaceae

Mabea fistulifera subsp. Mamoninha da mata Ar

bahiensis (Emmerich) Esser Mamoninha da mata A

$\begin{array}{cc}\text { Sapium argutum (Müll. } & \text { Borro leiteira }\end{array}$

Fabaceae

Albizia inundata (Mart.)

Barneby \& J. W. Grimes

Muquêm

$\mathrm{Ar}$

Anadenanthera peregrina

var. falcata (Benth.) Angico Me, Ma, $\mathrm{Ar}$ Altschul

Bauhinia forficata Link Pata de vaca Ar

Copaifera langsdorffii Desf Pau d'óleo Ar

Dimorphandra mollis Benth Faveira Ar

Dipteryx alata Vogel Barú Al, Ar

Inga edulis Mart. Ingá $\quad \mathrm{Al}, \mathrm{Ar}$

Inga ingoides (Rich.) Willd. Ingá de corda $\quad \mathrm{Al}, \mathrm{Ar}$

\section{Fabaceae}

Continua... 
Tabela 2. continuação.

\begin{tabular}{|c|c|c|}
\hline Famílias/espécie & Nome popular & *Uso \\
\hline Parkia platycephala Benth. & Fava de bolota & $\mathrm{Ar}$ \\
\hline $\begin{array}{l}\text { Piptadenia gonoacantha } \\
\text { (Mart.) J. F. Macbr. }\end{array}$ & Pau jacaré & $\mathrm{Ar}$ \\
\hline Platypodium elegans Vogel & Canzileiro & $\mathrm{Ar}$ \\
\hline Pterodon emarginatus Vogel & Sucupira & $\mathrm{Me}, \mathrm{Ar}$ \\
\hline $\begin{array}{l}\text { Schizolobium parahyba } \\
\text { (Vell.) Blake }\end{array}$ & Guapuruvu & $\mathrm{Ar}$ \\
\hline $\begin{array}{c}\text { Senegalia polyphylla (DC.) } \\
\text { Britton \& Rose }\end{array}$ & Monjoleiro & $\mathrm{Ar}$ \\
\hline $\begin{array}{c}\text { Tachigali vulgaris L.G.Silva } \\
\text { \& H. C. Lima }\end{array}$ & Cachamorra & $\mathrm{Ar}$ \\
\hline Tamarindus indica $\mathrm{L}$. & Tamarindo & $\mathrm{Al}, \mathrm{Ar}$ \\
\hline $\begin{array}{c}\text { Vatairea } \\
\text { macrocarpa (Benth.) Ducke }\end{array}$ & Angelim & $\mathrm{Ar}$ \\
\hline \multicolumn{3}{|l|}{ Lauraceae } \\
\hline $\begin{array}{l}\text { Persea americana } \\
\text { Mill. var. americana }\end{array}$ & Abacate & $\mathrm{Al}, \mathrm{Ar}$ \\
\hline $\begin{array}{l}\text { Physocalymma } \\
\text { scaberrimum Pohl }\end{array}$ & Cega machado & $\mathrm{Ar}$ \\
\hline \multicolumn{3}{|l|}{ Malpighiaceae } \\
\hline $\begin{array}{c}\text { Byrsonima coccolobifolia } \\
\text { Kunth }\end{array}$ & Murici rosa & $\mathrm{Al}, \mathrm{Co}, \mathrm{Ar}$ \\
\hline $\begin{array}{c}\text { Byrsonima crassifolia }(\mathrm{L} .) \\
\text { Kunth }\end{array}$ & Murici & $\mathrm{Al}, \mathrm{Co}, \mathrm{Ar}$ \\
\hline $\begin{array}{l}\text { Byrsonima pachyphylla A. } \\
\text { Juss. }\end{array}$ & Murici pequeno & $\mathrm{Al}, \mathrm{Co}, \mathrm{Ar}$ \\
\hline Malpighia glabra L. & Acerola & $\mathrm{Al}, \mathrm{Co}, \mathrm{Ar}$ \\
\hline \multicolumn{3}{|l|}{ Malvaceae } \\
\hline Apeiba tibourbou Aubl. & Escova de macaco & $\mathrm{Ar}$ \\
\hline Gossypium hirsutum L. & Algodão & $\mathrm{Ar}$ \\
\hline Guazuma ulmifolia Lam. & Mutamba & $\mathrm{Al}, \mathrm{Ar}$ \\
\hline $\begin{array}{c}\text { Luehea divaricata Mart. \& } \\
\text { Zucc. }\end{array}$ & Açoita cavalo & $\mathrm{Ar}$ \\
\hline $\begin{array}{l}\text { Sterculia striata A.St.-Hil. } \\
\text { \& Naudin }\end{array}$ & Xixá & $\mathrm{Ar}$ \\
\hline Theobroma cacao L. & Cacau & $\mathrm{Al}, \mathrm{Ar}$ \\
\hline $\begin{array}{l}\text { Theobroma grandiflorum } \\
\text { (Willd. ex Spreng.) K. } \\
\text { Schum. }\end{array}$ & Cupuaçú & $\mathrm{Al}, \mathrm{Co}$ \\
\hline \multicolumn{3}{|l|}{ Meliaceae } \\
\hline Azadirachta indica A. Juss. & Neem & $\mathrm{Ar}$ \\
\hline $\begin{array}{l}\text { Swietenia macrophylla } \\
\text { King }\end{array}$ & Mogno branco & $\mathrm{Ma}, \mathrm{Ar}$ \\
\hline \multicolumn{3}{|l|}{ Moraceae } \\
\hline $\begin{array}{l}\text { Artocarpus heterophyllus } \\
\text { Lam. }\end{array}$ & Jaca & $\mathrm{Al}, \mathrm{Ar}$ \\
\hline \multicolumn{3}{|l|}{ Musaceae } \\
\hline Musa paradisíaca $\mathrm{L}$. & Banana & $\mathrm{Al}, \mathrm{Co}, \mathrm{Ar}$ \\
\hline
\end{tabular}

Continua...
Tabela 2. continuação.

\begin{tabular}{|c|c|c|}
\hline Famílias/espécie & Nome popular & *Uso \\
\hline \multicolumn{3}{|l|}{ Myrtaceae } \\
\hline $\begin{array}{c}\text { Eugenia dysenterica (Mart.) } \\
\text { DC. }\end{array}$ & Caigata & $\mathrm{Al}, \mathrm{Me}, \mathrm{Ar}$ \\
\hline Psidium guajava $\mathrm{L}$ & Goiaba & $\mathrm{Al}, \mathrm{Ar}$ \\
\hline Psidium guyanense Pers. & Araçá & $\mathrm{Al}, \mathrm{Ar}$ \\
\hline \multicolumn{3}{|l|}{ Oxalidaceae } \\
\hline Averrhoa carambola L. & Carambola & $\mathrm{Al}, \mathrm{Ar}$ \\
\hline \multicolumn{3}{|l|}{ Rubiaceae } \\
\hline $\begin{array}{c}\text { Coffea canephora Pierre ex } \\
\text { A.Froehner }\end{array}$ & Café & $\mathrm{Al}, \mathrm{Ar}$ \\
\hline Genipa americana $\mathrm{L}$. & Genipapo & $\mathrm{Al}, \mathrm{Ar}$ \\
\hline Morinda citrifolia $\mathrm{L}$ & Noni & $\mathrm{Me}, \mathrm{Ar}$ \\
\hline \multicolumn{3}{|l|}{ Rutaceae } \\
\hline Citrus reticulata Blanco & Tangerina & $\mathrm{Al}, \mathrm{Ar}$ \\
\hline Citrus $x$ aurantium $\mathrm{L}$. & Laranja & $\mathrm{Al}, \mathrm{Ar}$ \\
\hline Citrus x limon (L.) Osbeck & Limão & $\mathrm{Al}, \mathrm{Ar}$ \\
\hline \multicolumn{3}{|l|}{ Sapindaceae } \\
\hline $\begin{array}{l}\text { Magonia pubescens A. } \\
\text { St.-Hil. }\end{array}$ & Tingui & $\mathrm{Ar}$ \\
\hline \multicolumn{3}{|l|}{ Simaroubaceae } \\
\hline $\begin{array}{c}\text { Simarouba versicolor A. } \\
\text { St.-Hil. }\end{array}$ & Mata menino & $\mathrm{Ar}$ \\
\hline \multicolumn{3}{|l|}{ Urticaceae } \\
\hline $\begin{array}{c}\text { Cecropia pachystachya } \\
\text { Trécul }\end{array}$ & Embaúba & $\mathrm{Ar}$ \\
\hline \multicolumn{3}{|l|}{ Verbenaceae } \\
\hline $\begin{array}{l}\text { Citharexylum myrianthum } \\
\text { Cham. }\end{array}$ & Tarumã branco & $\mathrm{Ar}$ \\
\hline \multicolumn{3}{|l|}{ Vochysiaceae } \\
\hline Qualea parviflora Mart. & Pau terra & $\mathrm{Ar}$ \\
\hline $\begin{array}{l}\text { Salvertia convallariodora } \\
\text { A. St.-Hil. }\end{array}$ & Colher de vaqueiro & $\mathrm{Ar}$ \\
\hline
\end{tabular}

Em que: * = espécies mais utilizadas pelos reassentados; $\mathrm{Al}=$ alimentação, $\mathrm{Ar}=$ arborização, $\mathrm{Ma}=$ madeira $\mathrm{Me}=$ medicinal $; \mathrm{Co}=$ comercial .

O índice de diversidade de Shannon-Wiener (H') nos quatro quintais agroflorestais, avaliados separadamente, variou de 2,52 a 3,27 e a equabilidade de Pielou (J') variou de 0,72 a 0,90 , demonstrando alta riqueza e diversidade, ou seja, baixa dominância ecológica e uma forte heterogeneidade ambiental (Tabela 3). 
Tabela 3. Índices de diversidade e equabilidade para os quintais agroflorestais amostrados no Reassentamento Mariana, TO.

\begin{tabular}{cccc}
\hline Áreas de estudo & $\mathbf{A}$ & $\mathbf{( H ' )}$ & (J') \\
\hline QAs Mariana* & 0,72 & 3,68 & 0,83 \\
QA 01 & 0,18 & 2,52 & 0,78 \\
QA 02 & 0,18 & 3,27 & 0,90 \\
QA 03 & 0,18 & 2,66 & 0,77 \\
QA 04 & 0,18 & 2,94 & 0,85 \\
\hline
\end{tabular}

Em que: A = área amostral (ha); H' = índice de diversidade de ShannonWeaver; J' = equabilidade de Pielou. Limite de inclusão = indivíduos com circunferência a $1,30 \mathrm{~m}$ do solo $\geq 10 \mathrm{~cm}$.

\section{Discussão}

Observou-se nos quintais agroflorestais (QAs) amostrados, no Reassentamento Mariana, TO, uma baixa porcentagem de espécies congêneres. De acordo com Webb (2000), as espécies congêneres geralmente competem pelos mesmos recursos e disputam nichos ecológicos semelhantes. Desta forma, é possível afirmar que essa pouca quantidade de espécies congêneres reflete uma grande interação ecológica dos quintais agroflorestais do Reassentamento Mariana e essa variedade de táxons contribui para o equilíbrio biológico desses espaços. Segundo os reassentados, a baixa quantidade de espécies congêneres é um resultado da procura pela maior variedade de plantas, uma vez que a escolha dos indivíduos e espécies inclusos no quintal é diretamente ligada ao uso das mesmas.

A família Fabaceae, que foi a mais rica em espécies, nos quintais estudados, se destaca não só no Cerrado, mas também em outras formações vegetais, como a Caatinga, Mata Atlântica e Amazônia. Considerada uma das maiores famílias de Angiosperma do globo, também foi destaque de outras pesquisas em QAs (Alves et al., 2013; Almeida et al., 2014). Essa predominância da família Fabaceae é resultado da seleção das espécies mantidas ou inseridas nos quintais pelos proprietários.

O sucesso adaptativo da família Fabaceae em áreas de Cerrado pode estar relacionado ao estabelecimento de relações simbióticas com microrganismos, responsáveis pelo aumento da capacidade de absorção de $\mathrm{P}$ e N, elementos encontrados em baixas concentrações nos solos oligotróficos do Domínio Cerrado e que são fundamentais para o crescimento e estabelecimento das espécies (Amaral et al., 2015). A importância dessa família no manejo e recuperação do solo é de conhecimento dos reassentados. Segundo os proprietários, no decorrer dos anos no reassentamento foram realizados cursos e visitas técnicas sobre o manejo de espécies e recuperação de áreas.

Conforme relatado pelos reassentados, a manutenção e inserção de algumas espécies da família Fabaceae ocorreu devido ao seu potencial de uso. A exemplo disso, espécies como o ingá (Inga sp.) e o tamarindo (Tamarindus indica) fazem parte da alimentação. Outras espécies, como o Angico (Anadenanthera peregrina) e a sucupira (Pterodon emarginatus) são utilizadas pelos proprietários tanto com fins não madeireiros, através de remédios caseiros, quanto pelo uso madeireiro, na construção de móveis e estrutura das residências.

As famílias Malvaceae, Arecaceae e Malpighiaceae também estão entre as principais famílias encontradas nos estudos de Gazel Filho (2008) e Pinto (2012). Essas famílias são bem representativas nos QAs da Amazônia, uma vez que são fontes de alimento e outros produtos utilizados como fonte de renda para os agricultores através, sobretudo, do beneficiamento dos frutos (Pinho, 2008; Vieira et al., 2012; Figueiredo Junior et al., 2013).

No reassentamento Mariana, uma das principais fontes de renda coletiva e familiar é a comercialização dos produtos, sejam eles in natura ou processados. Algumas espécies das famílias Malvaceae, Arecaceae e Malpighiaceae, como cupuaçu (Theobroma grandiflorum), açaí (Euterpe oleracea), murici (Byrsonima sp.) e acerola (Malpighia glabra), são comercializadas tanto in natura ou processadas, na forma de polpa ou doces.

No que se refere ao tamanho dos QAs do reassentamento Mariana, embora tenha sido delimitada a área das parcelas para o levantamento das espécies e indivíduos do estrato arbustivo e arbóreo em 0,18 ha para cada quintal, eles apresentavam tamanhos diferentes. Desta forma, vale ressaltar que esses QAs são encontrados nos mais diversos formatos e tamanhos. Conforme Nair (1986), os quintais agroflorestais são encontrados, geralmente, com menos de $10.000 \mathrm{~m}^{2}$. Corroborando com isso, os estudos já realizados em outros países apontam essa variação de tamanhos, como é o caso de Cuba, com QAs de 600 a $1.500 \mathrm{~m}^{2}$ (Wazel \& Bender, 2003); Indonésia, com 240 a $24.000 \mathrm{~m}^{2}$ (Kehlenbeck \& Maass, 2004); e na região de Machipanda, distrito de Monica em Moçambique, onde os quintais variam de $400 \mathrm{~m}^{2}$ a $5.000 \mathrm{~m}^{2}$ (Chitsondzo \& Silva, 2013). 
No Brasil, QAs também apresentam tamanhos muito variados. Florentino et al. (2007) estudaram os QAs no município de Caruaru, PE e encontraram quintais entre 140 a $12.500 \mathrm{~m}^{2}$, com média de $3.300 \mathrm{~m}^{2}$. Enquanto Gazel Filho (2008), estudando os QAs do município de Mazagão, AP, encontrou quintais entre 3.510 a 8.260 $\mathrm{m}^{2}$. Nos quintais indígenas de Araçá, RR, os mesmos variaram de 451 a $35.173 \mathrm{~m}^{2}$ (Pinho, 2008). Vieira et al. (2012), estudando os quintais do município de Bonito, PA, observaram tamanhos entre 500 e 2.500 $\mathrm{m}^{2}$ e Figueiredo Junior et al. (2013), em Anapu, PA, amostraram quintais com tamanhos entre 785 a 10.710 $\mathrm{m}^{2}$, em uma área do Projeto de Desenvolvimento Sustentável (PDS) Virola-Jatobá. Nos quintais do assentamento rural Santo Antônio, em Santarém, PA, o tamanho médio encontrado para os quintais foi de 720 $\mathrm{m}^{2}$ (Almeida \& Gama, 2014).

Os tamanhos e formatos dos quintais agroflorestais variam conforme a realidade ambiental da propriedade e a relação que os proprietários têm com os seus lotes, de acordo com a divisão das áreas de uso, de proteção, e de lazer. No reassentamento Mariana os lotes são divididos em pasto, residência, quintal, área de criação de animais, horta, lavoura e área de proteção permanente de hidrografias. De acordo com os reassentados, os quintais não têm limites físicos como cercas ou piquetes, mas há um limite conforme a divisão de uso da terra.

A média de indivíduos arbóreos encontrados nos quatro quintais estudados no reassentamento Mariana foi de 119; este quantitativo está próximo das médias obtidas em quintais agroflorestais amostrados em outras pesquisas (Pinto, 2012; Vieira et al., 2012, Figueiredo Junior et al., 2013).

Pinto (2012) encontrou 4.314 indivíduos, em um levantamento realizado em uma área equivalente a 3,84 ha, distribuída em 55 quintais, em bairros indígenas do município de São Gabriel da Cachoeira, AM, o que corresponde a uma média de 78 indivíduos por quintal. Vieira et al. (2012) estudaram a agrobiodiversidade de 24 quintais agroflorestais no município de Bonito, PA, com tamanho médio de 0,16 ha, e encontraram 1.168 indivíduos, obtendo desta forma, uma média de 48,6 indivíduos por quintal. Figueiredo Junior et al. (2013), ao caracterizarem a estrutura e a composição florística dos quintais agroflorestais do PDS Virola-Jatobá município de Anapu, PA, amostraram um somatório de 831 indivíduos referentes a 15 quintais agroflorestais, com tamanhos entre 0,078 ha a 1,07 ha; obtendo, desta forma, uma média de 55,4 indivíduos por quintal.
$\mathrm{O}$ número de indivíduos encontrados nos quintais do reassentamento Mariana está diretamente ligado ao histórico dos reassentados e de suas relações anteriores, quando ribeirinhos, em áreas adensadas por vegetação, e no manejo das mesmas, bem como pelo sentimento de pertencimento à terra e às novas formas de manejá-la adquiridas no decorrer dos anos. Além desse sentimento, a seleção e plantio de maior número de indivíduos de determinadas espécies, como o açaí (E. oleracea), o cajá (Spondias mombin) e a acerola (M. glabra) estão associados também ao fator econômico, para produção e comercialização de polpas.

De acordo com Ferreira \& Bonfim (2013), o processo de migração das pessoas para novos territórios tem como consequência a perda dos parâmetros de identidade da comunidade, o que de certa forma dificulta a adaptação às novas áreas. Porém, Melo \& Scopinho (2015) ressaltam que os assentamentos podem proporcionar, através de um processo coletivo, uma concepção de redes que sustentam os sentimentos de pertencimento e de restabelecimento de suas identidades.

Houve uma proximidade na quantidade das espécies levantadas entre os quatro quintais do reassentamento Mariana, sendo observado uma pequena diferença entre o valor mínimo (31 espécies) e o máximo (38 espécies). Essa aproximação no número de espécies entre os quintais demonstra que as famílias reassentadas se assemelham na relação sociocultural e no manejo dos quintais.

Das 81 espécies inventariadas nos quatro quintais, 44 são utilizadas diretamente pelas famílias proprietárias, sendo esse uso familiar ou comercial, como alimentar, medicinal ou madeireiro (Tabela 1). Dentre essas 44 espécies, três delas tem aproveitamento madeireiro, na construção de moveis, carroças e das casas e oito como remédio caseiro, sendo aproveitadas as cascas e folhas. Para alimentação, foram indicadas pelos reassentados 37 espécies frutíferas. As espécies com valor econômico e que são comercializadas in natura ou beneficiadas e processadas foram 12 , sendo que sete delas são utilizadas para a produção de polpas.

Apenas quatro espécies estiveram presentes em todos os Qas, sendo Anacardium occidentale, M. glabra, Psidium guajava e $S$. mombin. Essas espécies também foram bem representativas em outros levantamentos realizados em QAs (Florentino et al., 2007; Vieira et al., 2012; Chitsondzo \& Silva, 2013). A presença constante dessas espécies, tanto nos QAs do reassentamento Mariana, quanto nos quintais tropicais, está diretamente 
relacionada ao uso alimentar das mesmas, garantindo diversidade à dieta das famílias rurais (Pereira et al., 2010; Chitsondzo \& Silva, 2013; Figueiredo Junior et al., 2013).

Além de estarem diretamente relacionados com a segurança alimentar das famílias rurais, esses QAs são responsáveis também pela oferta de outros produtos e serviços, que contribuem com a diminuição de gastos financeiros dessas famílias, e que suprem grande parte de suas necessidades. Essa associação de áreas diversificadas, com o uso diário dos serviços, bem como o envolvimento financeiro, torna os quintais sustentáveis (Pinho, 2008; Chitsondzo \& Silva, 2013, Santos et al., 2016).

O índice de diversidade encontrado nos QAs do reassentamento Mariana $(03,68)$ corrobora com Gliessman (2001), que afirma que valores de diversidade de Shannon entre 3 e 4 são encontrados em ecossistemas naturais relativamente diversificados, ou seja, quanto maior o índice de diversidade em uma área estudada, maior será a complexidade e estabilidade, e menor será a variabilidade desse sistema (Freitas \& Magalhães, 2012; Alves et al., 2013). Ainda que não sejam ecossistemas naturais, Nair (1986) afirma que os quintais agroflorestais são considerados como um dos modelos mais antigos do uso da terra. De acordo com Kumar et al. (1994), têm sido perceptíveis em muitas situações a aproximação e harmonização desses espaços com ambientes naturais.

Os quintais do reassentamento Mariana apresentaram valores expressivos de equabilidade $(0,83)$. De acordo com Vieira et al. (2012), quanto mais o valor de equabilidade se aproxima de um, maior é a diversidade alcançável das espécies, representando desta forma uma boa distribuição do número de indivíduos por espécies.

De acordo com os reassentados, a proximidade do número de indivíduos por espécies foi estratégica, principalmente em espécies frutíferas, a fim de diversificar a produção alimentar. Além disso, todos os proprietários ressaltaram o sentimento de bem estar em tornar os quintais em ambientes multistratificados. Esse sentimento de bem estar devido à proximidade com a vegetação também é mencionado por outros proprietários de quintais agroflorestais do Brasil (Pinho, 2008; Miranda et al., 2012; Vieira et al., 2012).

Os valores médios para os índices de diversidade encontrados no reassentamento Mariana foram de $H^{\prime}=2,84$ e $J^{\prime}=0,82$. Há levantamentos em outros quintais agroflorestais brasileiros onde os pesquisadores avaliaram a diversidade e/ou a equabilidade dos estratos arbustivos e arbóreos e obtiveram valores médios próximos dos encontrado no reassentamento Mariana. Pinho (2008) obteve o valor médio de diversidade de 0,87 estudando 60 quintais indígenas de Araçá, RO e Vieira et al. (2012) encontraram em 24 quintais de Bonito, PA, uma média de 2,21 para diversidade e 0,85 para equabilidade. Em estudo em São Gabriel da Cachoeira, AM, Pinto (2012), encontrou valor médio de diversidade 1,73 e de equabilidade de 0,60 , estudando 55 quintais em bairros indígenas do município.

Os QA2 e QA4 alcançaram os maiores valores de diversidade e equabilidadade (Tabela 3), não havendo entre os dois quintais uma grande discrepância de indivíduos de uma única espécie. Apenas S. mombin (cajá) obteve mais que $10 \%$ dos indivíduos no QA2; $85,7 \%$ dos indivíduos encontrados do QA2 estão bem distribuídos entre as 37 espécies restantes. A abundância dessa espécie ocorre em função da introdução dessas plantas, por ser utilizada para produção de polpas. No QA4 as espécies Acrocomia aculeata (macaúba) e Cecropia pachystachya (embaúba) foram responsáveis por $28,9 \%$ dos indivíduos e $70,1 \%$ deles estão distribuídos entre as 29 espécies restantes. Conforme os proprietários do QA4, A. aculeata teve surgimento espontâneo e a família manteve os novos indivíduos, uma vez que a macaúba é utilizada para alimentação. A espécie $C$. pachystachya não tem um uso definido pela família. Porém, trata-se de espécie de fácil dispersão e de crescimento rápido, sendo mantida no quintal por comodidade.

Em relação aos QA1 e QA3, os índices de Shannon e de Pielou apresentaram menores valores que os outros dois quintais. Essa diferença pode ser explicada devido à maior quantidade de indivíduos por espécies. No QA1 as espécies E. oleracea e $P$. guajava foram responsáveis por $53,7 \%$ dos indivíduos amostrados. Conforme relatado pela família proprietária, E. oleracea é abundante devido à introdução dessas plantas para o manejo, uma vez que é utilizada para produção de polpas e faz parte da alimentação familiar. $\mathrm{O}$ mesmo principio foi utilizado para $P$. guajava, uma vez que é utilizada na alimentação e para a produção de doces. Enquanto no QA3, 44,7\% dos indivíduos foram representados por somente duas espécies Musa paradisiaca e M. glabra. A abundância de $M$. paradisiaca está diretamente ligada à alimentação da família, bem como à comercialização in natura. $M$. glabra também é manejada para alimentação e produção de polpas. 
Os valores do índice de Shannon (H') obtidos para os QAs do reassentamento Mariana variaram entre 2,52 QA1 e 3,27 QA2 (Tabela 3). Esses valores de diversidade foram bastante próximos aos valores encontrados por Gazel Filho (2008) nos quintais de Mazagão, PA (H'= 2,18 a 3,34). Na península de Kerala, na Índia, os valores de diversidade variaram de 1,29 a 3,02 (Kumar et al., 1994). Nos quintais indígenas de Araçá, RR a diversidade variou entre 0,26 e 1,18 (Pinho, 2008). Em quintais da comunidade Expedito Ribeiro, em Santa Bárbara do Pará, PA, os índices variaram entre 0,73 a 2,30 (Gonçalves et al., 2015) e nos quintais de Bonito, $\mathrm{PA}$, os valores encontrados variaram entre 1,60 e 2,77 (Vieira et al., 2012).

Os índices de Pielou (J') obtidos no reassentamento Mariana estiveram entre 0,77 no QA3 e 0,90 no QA2 (Tabela 3). Esses valores de equabilidade foram próximos aos encontrados por Vieira et al. (2012), que obtiveram valores entre 0,64 e 0,97, e por Gazel Filho (2008), que relataram valores variando de 0,67 a 0,8 .

Os valores de diversidade e equabilidade encontrados no reassentamento Mariana são considerados altos, refletindo uma grande diversidade de espécies e uma boa distribuição de indivíduos por espécie. Conforme Kumar et al. (1994), a alta diversidade dos quintais agroflorestais está associada a fatores socioeconômicos e culturais, de forma que esses influenciam na maneira como os proprietários distribuem e diversificam as espécies nesses espaços. Corroborando com isso, Semedo \& Barbosa (2007), afirmam que o conhecimento etnobotanico é o principal responsável pela diversidade dos quintais agroflorestais. Desta forma, é possível afirmar que a conservação das espécies, bem como a distribuição dos indivíduos dos quintais do reassentamento Mariana estão diretamente relacionados com a forma que os proprietários manejam essas áreas.

\section{Conclusões}

A diversidade e a riqueza das espécies arbustivasarbóreas dos quatro quintais agroflorestais (QAs) do reassentamento Mariana, no Estado do Tocantins, apresentaram altos valores, caracterizando, uma boa distribuição das espécies inventariadas. Os valores encontrados no reassentamento Mariana estão diretamente ligados ao manejo dessas áreas pelas famílias proprietárias.
Pode-se inferir que os QAs são importantes, tanto no âmbito ecológico, quanto no socioambiental, uma vez que refletem a capacidade dos proprietários de diversificarem esses espaços, preservarem espécies nativas e, consequentemente, contribuirem para a conservação da biodiversidade, bem como garantirem o bem estar das suas famílias.

Tendo em vista a relevância dos QAs e a escassez de informações referentes a essas áreas no estado do Tocantins, é possível apontar a necessidade de mais pesquisas e da formação de um banco de dados, com o intuito de reforçar o investimento de políticas públicas, por meio da extensão rural, dos órgãos ambientais e de saúde, uma vez que esses quintais contemplam a conservação da biodiversidade e são responsáveis pela segurança alimentar dos proprietários e das comunidades.

\section{Referências}

Albuquerque, U. P. et. al. Métodos e técnicas para coleta de dados etnobiológicos. In: Albuquerque, U. P. et al. (Ed.). Métodos e técnicas na pesquisa etnobiológica e etnoecológica. Recife: Núcleo Publicações em Ecologia e Etnobotânica Aplicada, 2010. p. 39-64.

Almeida, L. S. D. \& Gama, J. R. V. Quintais agroflorestais: estrutura, composição florística e aspectos socioambientais em área de assentamento rural na Amazônia brasileira. Ciência Florestal, v. 24, n. 4, p. 1041-1053, 2014. DOI: 10.5902/1980509816617.

Almeida, R. F. et al. Mudanças florística e estruturais no cerrado sensu stricto ao longo de 27 anos (1985-2012) na Fazenda Água Limpa, Brasília, DF. Revista Rodriguésia, v. 65, n. 1, p. 1-19, 2014. DOI: 10.1590/S2175-78602014000100001.

Alves, H. R. et al. Fitossociologia e grupos ecológicos da comunidade lenhosa em um remanescente de cerradão em Uberlândia, MG. Revista Caminhos de Geografia, v. 14, n. 46, p. 236-245, 2013.

Amaral, G. M. et al. Aspectos da distribuição de Mimosoideae (Fabaceae) arbóreas no planalto de Santa Catarina, sul do Brasil. Neotropical Biology and Conservation, v. 10, p. 74-84, 2015.

Angiosperm Phylogeny Group. An update of the Angiosperm Phylogeny Group classification for the orders and families of flowering plants: APG III. Botanical Journal of the Linnean Society, n. 161 , n. 2, p. 105-121, 2009. DOI: 10.1111/j.10958339.2009.00996.x.

Batista, E. A. D. A recomposição do modo de vida nos reassentamentos rurais do setor elétrico: estudo comparativo entre Flor da Serra e São Francisco de Assis (Estado do Tocantins). 2009. 231 f. Dissertação (Mestrado em Ciências do Ambiente) - Fundação Universidade Federal do Tocantins, Tocantins.

Brizídio, A. K. \& Nunes, R. O. Composição florística dos quintais nos Bairros Floresta e Texeirão na Cidade de Cacoal, Rondônia. Revista Científica Facimed, v. 2, p. 195-210, 2010. 
Chagas, J. C. N. et al. Importância dos quintais agroflorestais na conservação de plantas aromáticas e condimentares em duas comunidades de várzea no amazonas. In: ENCONTRO NACIONAL DA ANPPAS, 6., 2012, Belém, PA. Anais... Belém, PA: ANPPAS, 2012.

Chitsondzo, C. C. E. \& Silva, I. C. Quintais caseiros em Machipanda, distrito de Manica, Moçambique. Pesquisa Florestal Brasileira, v. 33, n. 74, p. 127-135, 2013. DOI: 10.4336/2013.pfb.33.74.413.

Evans, A. et al. Assessment of sustainability indicators for renewable energy technologies. Renewable and Sustainable Energy Reviews, v. 13, n. 5, p. 1082-1088, 2009. DOI: 10.1016/j.rser.2008.03.008.

Ferreira, K. P. M. \& Bonfim, Z. A. C. Juventude no semiárido nordestino: caminhos e descaminhos da emigração. In: Leite, J. F. \& Dimenstein, M. (Org.). Psicologia e contextos rurais. Natal: EDUFRN, 2013. p. 89-116.

Finger, Z. \& Finger, F. A. Fitossociologia de comunidades arbóreas remanescentes de cerrado sensu stricto no Brasil Central. Floresta, v. 45, n. 4, p. 769-780, 2015. DOI: 10.5380/rf.v45i4.30860.

Figueiredo Junior, O. et al. Levantamento florístico dos quintais agroflorestais do PDS Virola Jatobá em Anapú, Pará. Enciclopédia Biosfera, v. 9, p. 1793-1805, 2013.

Florentino, A. T. N. et al. Contribuição de quintais agroflorestais na conservação de plantas da caatinga, Município de Caruaru, PE, Brasil. Acta Botanica, v. 21, n. 1, p. 37-47, 2007. DOI: 10.1590/ S0102-33062007000100005.

Freitas, E. P. et al. Delimitação de bacia hidrográfica no ambiente Google Earth. Irriga, v. 1, n. 1, p. 97-104, 2012.

Freitas, W. K. de \& Magalhaes, L. M. S. Métodos e parâmetros para estudo da vegetação com ênfase no estrato arbóreo. Floresta e Ambiente, v. 19, n. 4, p. 520-539, 2012. DOI: 10.4322/ floram.2012.054.

Gazel Filho, A. B. Composição, estrutura e função de quintais agroflorestais no Município de Mazagão, Amapá, Belém. 2008. 104 f. Tese (Doutorado em Ciências Agrárias) - Universidade Federal Rural da Amazônia, Belém, PA.

Gliessman, S. R. Diversidade e estabilidade do agroecossistema. In: Agroecologia: processos ecológicos em agricultura sustentável. Porto Alegre: Ed da UFRGS, 2001. 474 p.

Gonçalves, J. P. et al. Análise florística e estrutural de quintais agroflorestais na comunidade Expedito Ribeiro em Santa Bárbara do Pará. Enciclopédia Biosfera, v. 11, p. 173-183, 2015.

Gotelli, N. J. \& Ellison, A. M. Princípios de estatística em ecologia. Porto Alegre: Artmed, 2011.

Kehlenbeck, K. \& Maass, B. L. Crop diversity and classification of homegardens in Central Sulawesi, Indonesia. Agroforestry Systems, v. 63, n. 1, p. 53-62, 2004. DOI: 10.1023/B:AG FO.0000049433.95038.25.

Klink, C. A. \& Machado, R. B. A conservação do Cerrado brasileiro. Megadiversidade, v. 1, n. 1, p. 148-155, 2005.

Kumar, B. M. et al. Diversity, structure and standing stock of wood in the homegardens of Kerala in peninsular India. Agroforestry Systems, v. 25, n. 3, p. 243-262, 1994. DOI: 10.1007/BF00707463.
Kunz, S. H. et al. Estrutura fitossociológica de um cerradão em Canarana, estado do Mato Grosso, Brasil. Revista Acta Scientiarum Biological Sciences, v. 31, n. 3, p. 255-261, 2009. DOI: 10.4025/ actascibiolsci.v31i3.1625.

Leturcq, G. Migrations forçées dans le Sud du Brésil: les atingidos. 2010. 406 f. Thèse (Doctorat de Géographie) - Université du Maine, Le Mans, França.

Lorenzi, H. Árvores brasileiras: manual de identificação e cultivo de plantas arbóreas do Brasil. São Paulo: Instituto Plantarum, 2002.

Melo, T. G. \& Scopinho, R. A. Participação em cooperativas de assentamentos rurais: estudo sobre os sentidos do trabalho. Psicologia em Estudo, v. 20, p. 529-541, 2015. DOI: 10.4025/psicolestud. v20i4.25776.

Miguel, E. P. et al. Floristic-structural characterization and successional group of tree species in the Cerrado biome of Tocantins state, Brazil. Revista Caatinga, v. 29, n. 2, p. 393-404, 2016. DOI: 10.1590/1983-21252016v29n216rc.

Miranda, R. S. et al. Quintais agroflorestais como estratégia alimentar familiar no Assentamento 26 de Março, Marabá, Pará. Agroecossistemas, v. 4, n. 1, p. 68-80, 2012. DOI: 10.18542/ragros. v4i1.1051.

Nair, P. K. P. An evaluation of the struture and function of tropical homegardens. Agricultural Systems, v. 21, n. 4, p. 279-310, 1986. DOI: 10.1016/0308-521X(86)90104-6.

Parente, T. G. \& Miranda, C. M. Impactos socioculturais e gênero nos reassentamentos da Usina Luis Eduardo Magalhães - TO. Varia Historia, v. 30 , n. 53, p. 557-570, 2014. DOI: 10.1590/S010487752014000200011.

Pereira, C. N. et al. Caracterização de quintais agroflorestais no projeto de assentamento Belo Horizonte I, São Domingos do Araguaia, Pará. Agroecossistemas, v. 2, n. 1, p. 73-81, 2010. DOI: 10.18542/ragros.v2i1.1225.

Pinho, R. C. Quintais agroflorestais indígenas em área de savana (lavrado) na terra indígena Araçá, Roraima. 2008. 108 f. Dissertação (Mestrado em Ciências de Florestas Tropicais) Instituto Nacional de Pesquisas da Amazônia; Universidade Federal do Amazonas, Manaus.

Pinto, I. C. Agrobiodiversidade de quintais agroflorestais urbanos e perfil social de etnias indígenas em São Gabriel da Cachoeira, AM. 2012. 196 f. Tese. (Doutorado em Ciências Florestais) Universidade Federal de Lavras, Lavras.

Reflora: Herbário Virtual. Lista de espécies da flora do Brasil. Rio de Janeiro: Jardim Botânico do Rio de Janeiro, [2015]. Disponível em: <http://floradobrasil.jbrj.gov.br/2012/>. Acesso em: 23 ago. 2015.

Rocha Garcia, B. N. et al. Quintais agroflorestais e segurança alimentar em uma comunidade rural na Amazônia Oriental. Revista de la Facultad de Agronomia, n. 114, p. 67-73, 2015.

Sano, E. E. et al. Land cover mapping of the tropical savanna region in Brazil. Environmental Monitoringand Assessment, v. 166, n. 1-4, p. 113-114, 2010. DOI: 10.1007/s10661-009-0988-4. 
Santos, D. L. Solos do assentamento Mariana - Palmas-TO: características e aptidões. In: JORNADA DE INICIAÇÃO CIENTÍFICA, 9., 2009, Palmas. Anais... Palmas: Ceulp/Ulbra, 2009.

Santos, I. G. et al. Quintais agroflorestais na percepção dos moradores do reassentamento Mariana, Tocantins. Revista Verde de Agroecologia e Desenvolvimento Sustentável, v. 11, n. 5, p. 95-102, 2016.

Semedo, R. J. C. G. \& Barbosa, R. I. Árvores frutíferas nos quintais urbanos de Boa Vista, Roraima, Amazônia brasileira. Acta Amazonica, v. 37, n. 4, p. 497-504, 2007. DOI: 10.1590/S004459672007000400003.

Sheperd, G. J. Fitopac 2: manual do usuário. Campinas: Unicamp, $2010.91 \mathrm{p}$.

Silva Júnior, M. C. 100 árvores do cerrado: sentido restrito: guia de campo. Brasília, DF: Ed. Rede de Sementes do Cerrado, 2012. 304 p.
Silva, L. R. \& Sano, E. E. Análise das imagens do satélite RapidEye para discriminação da cobertura vegetal do bioma Cerrado. RBC. Revista Brasileira de Cartografia, v. 68, p. 1269-1283, 2016.

Tocantins. Secretaria do Planejamento e da Modernização da Gestão Pública. Atlas do Tocantins: subsídios ao planejamento da gestão territorial. 6. ed. Palmas, 2012. 80 p.

Vieira, T. A. et al. Agrobiodiversidade de quintais agroflorestais no município de Bonito, Estado do Pará. Revista de Ciências Agrárias, v. 55, n. 3 , p. $159-166,2012$.

Wazel, A. \& Bender, S. Plant species diversity of homegardens of Cuba and its significance for household food supply. Agroforestry systems, v. 57, n. 1, p. 39-49, 2003. DOI: 10.1023/A:1022973912195.

Webb, C. O. Exploring the phylogenetic structure of ecological communities: an example for rain forest trees. American Naturalist, v. 156 , p. $145-155,2000$. DOI: 10.1086/303378. 